IAC-14,C2,2,4,x23194

\title{
IMPORTANCE OF STRUCTURAL DAMPING IN THE DYNAMIC ANALYSIS OF COMPLIANT DEPLOYABLE STRUCTURES
}

\author{
Florence Dewalque \\ Department of Aerospace and Mechanical Engineering (LTAS), University of Liège, Belgium, f. dewalque@ulg.ac.be \\ Pierre Rochus, Centre Spatial de Liège, University of Liège, Belgium, prochus@ulg.ac.be \\ Olivier Brüls, LTAS, University of Liège, Belgium, o.bruls@ulg.ac.be
}

\begin{abstract}
Compliant mechanisms such as tape springs are often used on satellites to deploy appendices, e.g. solar panels, antennas, telescopes and solar sails. Their main advantage comes from the fact that their motion results from the elastic deformation of structural components, unlike usual hinges or prismatic joints, the motion of which is dictated by contact surfaces. No actuators or external energy sources are required and the deployment is purely passive, which appears as a decisive feature for the design of low-cost missions with small satellites or cubesats. The mechanical behaviour of a tape spring is intrinsically complex and nonlinear involving buckling, hysteresis and self-locking phenomena. High-fidelity mechanical models are then needed to get a detailed understanding of the deployment process, improve the design and predict the actual behaviour in the space 0-g environment. In the majority of the previous works, dynamic simulations were performed without any physical representation of the structural damping. These simulations could be successfully achieved because of the presence of numerical damping in the transient solver. However, in this case, the dynamic response turns out to be quite sensitive to the amount of numerical dissipation, so that the predictive capabilities of the model are questionable. In this work based on numerical case studies, we show that the dynamic simulation of a tape spring can be made less sensitive to numerical parameters when the structural dissipation is taken into account.
\end{abstract}

\section{INTRODUCTION}

With the extensive development of small satellites and cubesats dedicated to low-cost missions, the mass reduction of the components is paramount. However, the power reduction due to the miniaturisation of electronic equipment does not follow the same downward slope. Thus, covering only the external surface of the satellite with solar cells might not provide enough power and be too restrictive, hence the necessity for a reliable but cheap and simple means to deploy large solar panels. This, however, brings another major problem that must be solved: the packaging of large structures into the confined space inside the fairing of launch vehicles. In order to address these challenges, deployable structures have been developed and a brief listing of the most common structures can be found in [1]. This paper will focus on those belonging to the compliant mechanisms category and in particular on tape springs.

A tape spring is a thin strip curved along its width commonly known as a Carpenter tape and used in the everyday life as tape measures. Nowadays, it finds applications in the space domain for the deployment of appendices such as solar panels, antennas, telescopes and solar sails. Since they belong to the category of compli- ant mechanisms, they rely only on elastic energy that is stored during the folding and then naturally released when deployed. Indeed, a possible equilibrium state when the tape spring is free of constraints is the straight configuration, which is sought as a deployed configuration for many space applications. This characteristic also brings forward the fact that no source of external energy is required for the deployment and hence the passive and selfactuated behaviours of these devices.

Their motion results from the deformation of structural components only and not from the sliding between contact surfaces as in usual hinges or prismatic joints. It implies that tape springs do not require any lubricant to ensure a motion without jamming, which is advantageous in order to avoid outgassing and contamination in space. The structural simplicity of tape springs is also beneficial, since, without taking into account the supports, a tape spring consists of a single component and thus does not require any assembly procedure. The robustness is then greatly improved compared to more common mechanisms and it limits the possibilities of failure during deployment.

Regarding the mechanical behaviour of tape springs, it is complex and highly nonlinear, but stays in the elastic regime for the most commonly used materials such as 
$\mathrm{BeCu}$. Regarding other materials, a limit that the ratio between the transverse radius and the thickness must satisfy can be found in [2] to avoid any plastic deformation during folding.

The complexity is further increased due to the existence of nonlinear phenomena such as buckling which induces the formation of a fold in the deformed configuration and the non-superposition of the loading and unloading paths leading to hysteresis and self-locking.

The mathematical background describing the theoretical relationship between the bending angle forced at the extremities of the tape spring and the resulting bending moment was first developed by the following authors. Chronologically, Wüst [3] was the first to use equilibrium considerations starting from the theory of linear shell structures. Then, Rimrott [4] managed to obtain the same results through energetic considerations. Finally, Mansfield [5] created his own model thanks to a variational method and, most importantly, was the first one to take into account the torsional behaviour of the structure.

More recently, new analytical models were developed by several authors and usually combined to numerical studies and experiments for validation. In [6], the bending moment-angle relationship was numerically computed for a large variety of tape springs. It led to empirical expressions for the key parameters describing the behaviour of a tape spring and it was exploited to model and experimentally perform the deployment of a rigid panel fixed by two pairs of tape springs. In a next work [7], the dynamic deployment was investigated for two particular configurations: coiled around a circular hub and folded twice (first in opposite sense then in equal sense bending). It was shown that in the first case, an analytical model can be derived from conservative energy considerations, while in the second case, a non-conservative impulse-momentum formulation is required and allows representing a folded tape spring as two rigid bodies of variable length connected to each other with a mobile hinge. Between this 2D discrete model and finite element simulations with shells, an intermediate model was developed in [8] based on 1D planar rods with a flexible cross-section. Quasistatic analyses of the folded configuration when submitted to end loads were also performed in [9] and correctly predicted thanks to a variational technique.

So far, the different papers mentioned above focused on the folding and deployment of tape springs in two dimensions, that is with the fold line perpendicular to the longitudinal axis of the structure. To reach a more compact packaging, three dimensional foldings were analysed, first analytically in [10], then experimentally in $[11,12]$.
Tape springs can also be combined to form tape spring hinges. Several examples can be found in [13] under the form of tetrahedral structures and in [14] as pairs characterised by 2D and 3D folds. A generic hinge called MAEVA was developed by the CNES and 01dBMetravib [15] and was successfully used to deploy solar panels, antennas and masts on the six MYRIADE microsatellites [16]. Experimental analyses where performed, along with finite element simulations. Recently, a more precise study of the dynamic behaviour and the selflocking phenomenon was carried out in [17].

In the majority of the previous works, finite element dynamic simulations of tape springs were performed without any physical representation of the structural damping, with the notable exceptions of $[18,19]$. In the former, the viscoelastic material properties of a reinforced polymer are integrated in the finite element model under the form of a Prony series. The goal was to determine the impact of the temperature, the folding rate, the creep recovery and the relaxation during stowage on the behaviour of the tape spring. On the other hand, [19] does not directly concern tape springs, but analyses foldable flattenable tubes which are also characterised by buckling and hysteresis phenomena. The structural damping is in this case based on the Rayleigh model with the particularity that the damping factor is variable according to the state of the structure. Otherwise, the simulations could be successfully achieved because of the presence of numerical damping in the transient solvers. However, as it will be proven further in this paper, the dynamic response turns out to be quite sensitive to the amount of numerical dissipation. The predictive capabilities of the models are then questionable. Furthermore, in the analytical model developed in [7], it was shown that viscous damping terms needed to be introduced in order to match the numerical results to the experiments. The aim of this work is then to determine if adding structural damping can make the simulator less sensitive and if numerical damping is always required.

The layout of this paper is as follows. In Section II, the geometric characteristics of a tape spring are described. Then in Section III, the general features of the finite element models and the resolution strategy are explained. In Section IV, the theory ruling the bending behaviour of tape springs is recalled. In Section V, the effect of numerical and structural dampings in the generalized- $\alpha$ method is analysed on a one-degree-of-freedom model and compared to the analytical solution. In Section VI, the impact of the numerical damping on the bending behaviour of a tape spring is studied in the case of a dynamic deploy- 
ment. In Section VII, structural damping is added in the same model. Finally, the conclusions of this work are drawn in Section VIII.

\section{GEOMETRIC CHARACTERISTICS}

The geometry of a tape spring is defined by the means of five parameters: its length $L$, its thickness $t$, its subtended angle $\alpha$, its transverse radius of curvature $R$ and its longitudinal radius of curvature $R_{L}$. All these elements are represented in Figure 1.

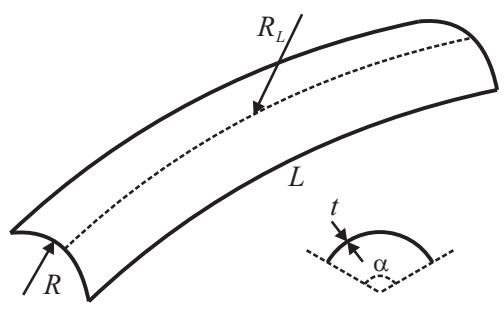

Figure 1: Geometric characteristics of a tape spring with the length $L$, the thickness $t$, the subtended angle $\alpha$, the transverse radius of curvature $R$ and the longitudinal radius of curvature $R_{L}$.

For the most common applications and the structures studied in this paper, the tape springs are straight without any longitudinal curvature. In other cases, they would be referred to as curved tape springs [20].

Regarding the ratio between the length and the width, a lower limit of five will be respected in order to reduce the local end effects due to the boundary conditions [5].

\section{FINITE ELEMENT MODELS}

All the finite element models in this paper are developed in the commercial software SAMCEF [21]. Since the thickness of a tape spring can be several orders of magnitude smaller than its other dimensions, the finite elements are linear shells based on the Mindlin-Reissner theory available in the software. Parabolic shells were also tested, but they did not have a significant impact on the results and the computational cost was unnecessarily increased.

Regarding the size of the mesh, a campaign of tests led to the choice of elements of $3 \mathrm{~mm}$ as the default size, for which all the key parameters describing a tape spring's behaviour have converged. However, in some particular cases, another size might be used and will be clearly specified.

Furthermore, nodes are added at the centroids of the cross-sections located at the extremities of the tape spring to which they are rigidly connected along the whole width. Their purpose is to give an easy means to control the angle of bending, to apply the boundary conditions and to determine the resulting bending moment.

The problems will be solved in two consecutive steps. First, starting from the equilibrium state corresponding to the straight configuration, the tape spring is folded to the required bending angle by performing a very slow dynamic analysis. Although static analyses taking the form of continuation methods can be exploited in this first part, the algorithm is quite sensitive to the parameters and severely increases the computation cost. As a consequence, they are not used in this work. A comparison between the static and dynamic results can be found in [17], which shows that the two approaches give very close results. Then, once the deformed configuration is stabilised, the structure is released and simulated with a standard dynamic analysis. In both situations, the following time step algorithms can be exploited: Newmark [22] of first-order accuracy, HHT [23] and generalized- $\alpha$ [24] both of second-order accuracy. However, only the former and the latter will be used in this work since it was observed that the HHT and generalized- $\alpha$ methods give similar results for the bending of tape springs [17]. Regarding the time step, an adaptive method is exploited in order to accurately capture the nonlinear phenomena and reduce the computation costs when the deformed configuration does not significantly evolve.

\section{BENDING BEHAVIOUR OF A TAPE SPRING}

The bending behaviour of a tape spring is direction dependent. The senses of bending were first defined by Wüst [3] and are represented in Figure 2 where the sign conventions for the bending moment $M$ and the bending angle $\theta$ are also visible.

The opposite sense bending is characterised by longitudinal and transverse curvatures in opposite sense. It induces tensile stresses along the edges and only bending efforts appear during the deformation.

On the contrary, for the equal sense bending, the longitudinal and transverse curvatures are in the same sense and it induces compressive stresses along the edges. However, the deformed configuration is characterised by flexural-torsional modes, thus bringing forward the complex behaviour resulting from this sense of bending. 

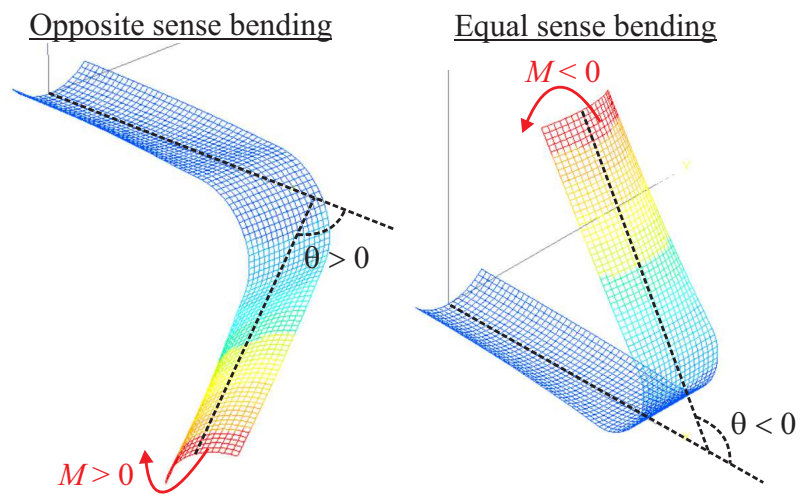

Figure 2: Senses of bending and sign conventions with $M$ the bending moment and $\theta$ the bending angle.

The evolution of the deformed configuration of a tape spring when submitted to opposite or equal sense bending and for the folding or the deployment of the structure can be explained with the help of the schematic diagram in Figure 3, which gives the corresponding bending moment when the tape spring is controlled in rotation through the bending angle.

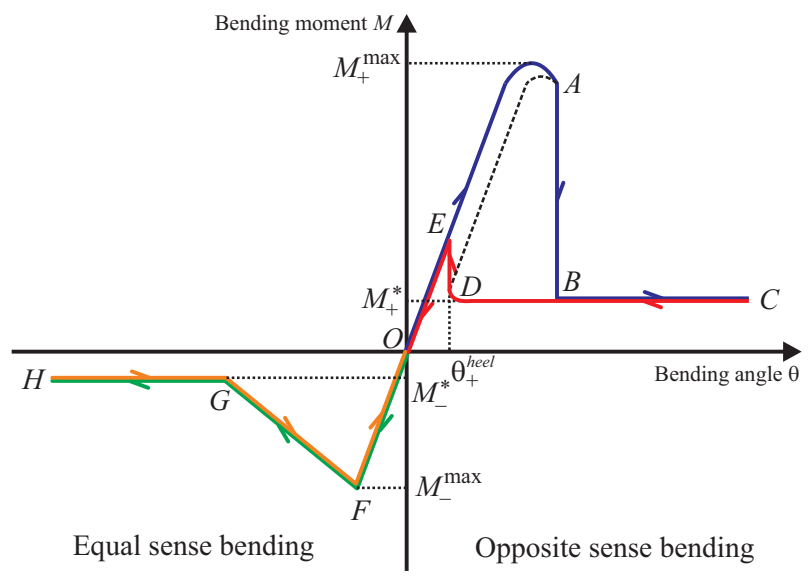

Figure 3: Bending moment-angle diagram. Blue $(O A B C)$ : loading in opposite sense; red $(C D E O)$ : unloading in opposite sense; green $(O F G H)$ : loading in equal sense; orange (HGFO): unloading in equal sense; broken line $(A D)$ : unstable path.

Starting from the origin $O$ and in the opposite sense, the bending moment first varies linearly with the bending angle for small rotations. The associated deformed configuration corresponds to a tape spring bended into a smooth curve. Then, for larger rotations, the increasing $M$ forces the cross section to flatten, especially in the middle, until it reaches a maximum: the peak moment $M_{+}^{\max }$.
Right after this critical value, the structure is submitted to buckling and a fold forms in the middle. The strains are then localized in this zone which is characterised by a uniform non-zero longitudinal curvature and a zero transverse curvature, while away from the fold region, the tape is practically straight. This buckling is accompanied by an important decrease in the bending moment represented on the diagram by the vertical line $A B$. In the particular case where the unstable equilibrium can be captured, the path defined by the curve $A D$ is followed. If the rotation between the extremities is further increased $(B C)$, the bending moment remains approximately constant to $M_{+}^{*}$ called the steady-state moment. In the meantime, the arc-length of the fold increases, but its longitudinal curvature does not vary. Regarding its value, it can be theoretically proven that it is equal to the initial transverse curvature of the tape spring [3,25]. Indeed, since the initial and deformed configurations are both cylindrical surfaces, the Gaussian curvature does not vary, resulting in the absence of stretching strain energy. If the bending strain energy is then minimised, it can be shown that the previous statement is valid. However, finite element studies proved that a difference exists between the two values, but it is marginal [2].

In the reverse direction which represents the deployment of the tape spring, the same path as for the folding is first followed $(C B)$. At point $B$, the bending moment does not go back to point $A$, but stays constant until it reaches point $D$ called the heel. There, the curve jumps to point $E$ where one can notice that the associated bending moment is smaller than the peak moment $M_{+}^{\max }$. For the last part $(E O)$, the linear behaviour is recovered.

The difference between the paths for the folding and the deployment is responsible for the hysteresis phenomenon, which itself, through the dissipation of energy, will lead to the self-locking of the tape spring.

For the equal sense bending and small rotations, $M$ also varies linearly with $\theta$ as in the opposite sense. Furthermore, the slope of $O F$ is equal to the slope of $O E$. However, in this case, the linear behaviour ends up much sooner. Buckling happens at point $\mathrm{F}$ for another critical value such that $\left|M_{-}^{\max }\right|<\left|M_{+}^{\max }\right|$. The structure is then less stiff when bended in this sense. The deformed shape of the tape spring is due to a coupling between bending and torsion efforts creating flexural-torsional modes. Near the extremities, asymmetric torsional folds increase in amplitude as the tape spring is folded and move towards its middle. At the same time, the bending moment decreases $(F G)$. When the folds reach the middle, they combine themselves to form a symmetric one as the one 
described in opposite sense. Then, for the rest of the folding $(G H)$, the bending moment remains constant $\left(M_{-}^{*}\right)$.

Finally, in equal sense bending, it is commonly assumed that the deployment path is superimposed on the folding path.

\section{IV.I Analysis with SAMCEF}

In order to show that the commercial software SAMCEF is able to capture the key parameters of a tape spring's behaviour, the structure with the geometric and material characteristics specified in Table 1 is analysed.

\begin{tabular}{cccc}
\hline$L$ & $t$ & $\alpha$ & $R$ \\
\hline $200 \mathrm{~mm}$ & $0.1 \mathrm{~mm}$ & $90^{\circ}$ & $20 \mathrm{~mm}$ \\
\hline \hline$E$ & $v$ & $\rho$ \\
\hline $131000 \mathrm{MPa}$ & 0.3 & $8100 \mathrm{~kg} / \mathrm{m}^{3}$ \\
\hline
\end{tabular}

Table 1: Geometric and material characteristics of the tape spring analysed in SAMCEF and [6].

To perform the simulation, one of the nodes located at the centroids is clamped, while on the other one, the bending angle is controlled and the displacements in the transverse direction are locked in order to guide the deformation without restraining the torsion. The mesh size is fixed to $1 \mathrm{~mm}$. Regarding the time integration scheme, the Newmark method [22] is chosen with a numerical damping defined by the spectral radius and equal to zero in order to annihilate the oscillations after the buckling in a minimum number of time steps. The results are given in Figure 4 where the artificial vertical peaks occurring during the buckling phenomena are due to the transient behaviour of the solution.

For the loading in the opposite sense (blue o-curve), all the characteristic features are well represented by the model: the linear behaviour for small rotations, the peak moment $M_{+}^{\max }$ followed by the buckling of the structure and a steady-state moment $M_{+}^{*}$. During the unloading (red $\square$-curve), the bending moment jumps back on the loading curve for a smaller bending angle than the one at which buckling occurred. Thus the hysteresis phenomenon is well captured.

Regarding the loading (green $\triangle$-curve) and unloading (black +-curve) paths in the equal sense, they are almost identical and are characterised by a peak moment $M_{-}^{\max }$ and a steady-state moment $M_{-}^{*}$ for larger rotations. Furthermore, the slope in the linear zone is equivalent to the one created in opposite sense bending. As it was mentioned before, the equal sense bending leads to a more complex behaviour due to flexural-torsional modes. It

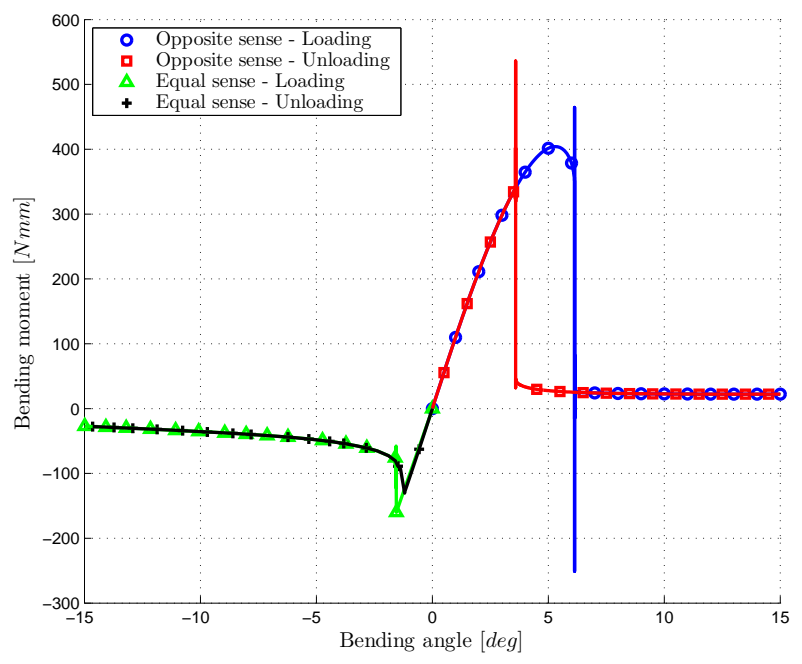

Figure 4: Bending moment-angle behaviour from SAMCEF with the characteristics of Table 1 .

results in a series of disruptive oscillations in the $M(\theta)$ curve whose amplitude increases with the mesh size (Figure 5). However, the mean behaviour of the curve associated to a mesh size of $2 \mathrm{~mm}$ still matches the reference curve obtained with a mesh size of $1 \mathrm{~mm}$. Notice that the peak moment $M_{-}^{\max }$ is truncated on the plot in order to focus on these oscillations.

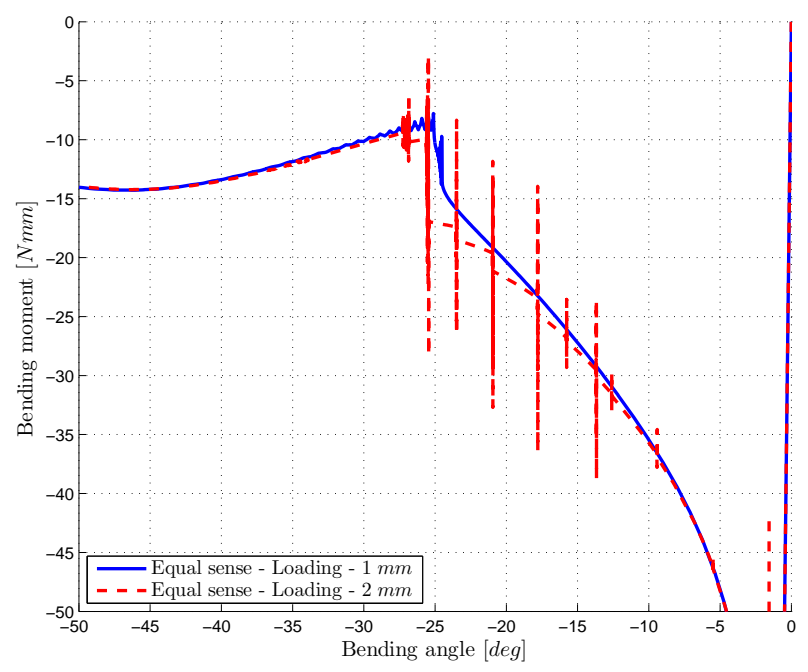

Figure 5: Vertical zoom on the loading part in equal sense bending form the mesh sizes $1 \mathrm{~mm}$ and $2 \mathrm{~mm}$.

Regarding the quantitative accuracy of the software, the results displayed in Figure 4 fairly reproduce those nu- 
merically obtained by Seffen in [6]. Compared to experimental investigations in [11] on two dimensional folds with the characteristics of Table 2, the average peak moment and steady-state moment in opposite sense bending are obtained with a difference of $5.34 \%$ and $1.91 \%$ respectively. However, for smaller lengths, the correlation for the peak moment is far less effective due to end effects. Indeed, Seffen [7] showed by comparing analytical, experimental and numerical results for a same tape spring that the peak moment can strongly differ for short tape springs, while the steady-state moment is not affected.

\begin{tabular}{|c|c|c|c|}
\hline$L$ & $t$ & $\alpha$ & $R$ \\
\hline $433 \mathrm{~mm}$ & $0.11459 \mathrm{~mm}$ & $1.719 \mathrm{rad}$ & $15.37 \mathrm{~mm}$ \\
\hline & $E$ & $\rho$ & \\
\hline & $95300 \mathrm{MPa}$ & $8100 \mathrm{~kg}$ & $m^{3}$ \\
\hline
\end{tabular}

Table 2: Geometric and material characteristics of the tape spring experimentally analysed in [11].

\section{$V$ DISSIPATION IN THE GENERALIZED- $\alpha$ METHOD}

In order to have a better understanding of the influence of structural and numerical dampings in the most common time integration schemes (Newmark, HHT, generalized$\alpha$ ), a simple example will be studied: the mass-springdamper system (Figure 6). All these methods are characterised by a controllable numerical damping, the first goal of which is to take part in the dissipation of high frequencies. Indeed, when a structure is discretized by the means of a finite element model, the high frequency modes are poorly represented and must be filtered in order to improve the quality of the system's response, while the low frequency modes must remain unaffected by the damping. Furthermore, the introduction of numerical damping in these methods ensures a better convergence of the algorithm, especially in the case of nonlinear systems.

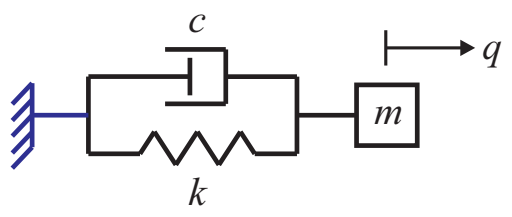

Figure 6: Mass $m$-spring $k$-damper $c$ system.

It can be easily shown that the Newmark and HHT methods are particular cases of the generalized- $\alpha$ method. This latter will then be used in the next developments and the specific values of the parameters to recover the other methods will be recalled.
The generalized- $\alpha$ method relies on the following update formulae [24]:

$$
\begin{array}{r}
\mathbf{M}\left(\mathbf{q}_{n+1}\right) \ddot{\mathbf{q}}_{n+1}+\mathbf{g}\left(\mathbf{q}_{n+1}, \dot{\mathbf{q}}_{n+1}, t_{n+1}\right)=0 \\
\mathbf{q}_{n+1}=\mathbf{q}_{n}+h \dot{\mathbf{q}}_{n}+h^{2}(0.5-\beta) \mathbf{a}_{n}+h^{2} \beta \mathbf{a}_{n+1} \\
h \dot{\mathbf{q}}_{n+1}=h \dot{\mathbf{q}}_{n}+h^{2}(1-\gamma) \mathbf{a}_{n}+h^{2} \gamma \mathbf{a}_{n+1} \\
\left(1-\alpha_{m}\right) h^{2} \mathbf{a}_{n+1}+\alpha_{m} h^{2} \mathbf{a}_{n}=\left(1-\alpha_{f}\right) h^{2} \ddot{\mathbf{q}}_{n+1}+\alpha_{f} h^{2} \ddot{\mathbf{q}}_{n}
\end{array}
$$

where the first equation is a general equation of motion, $\mathbf{q}$ is the vector of degrees of freedom, $\mathbf{M}$ is the mass matrix, $\mathbf{g}$ is the vector of external forces, $t$ is the time, $h$ is the time step, a is an acceleration-like variable with no particular physical meaning and $\beta, \gamma, \alpha_{m}, \alpha_{f}$ are numerical parameters. The Newmark method is recovered when $\alpha_{f}=\alpha_{m}=0$, while the HHT method requires to have $\alpha_{m}=0, \alpha_{f} \in[0,1 / 3], \gamma=0.5+\alpha_{f}$ and $\beta=0.25(\gamma+0.5)^{2}$.

For the generalized- $\alpha$ method, the numerical damping is controlled through the spectral radius at infinite frequencies $\rho_{\infty}$ which belongs to the interval $[0,1]$ according to:

$$
\alpha_{m}=\frac{2 \rho_{\infty}-1}{\rho_{\infty}+1} \quad \text { and } \quad \alpha_{f}=\frac{\rho_{\infty}}{\rho_{\infty}+1}
$$

When $\rho_{\infty}=0$, the dissipation leads to a complete annihilation of the high frequency content and, on the opposite, when $\rho_{\infty}=1$ there is no dissipation.

In order to assess the level of dissipation, the spectral radius is used. It is defined as:

$$
\rho=\max \left(\left|\lambda_{1}\right|,\left|\lambda_{2}\right|,\left|\lambda_{3}\right|\right)
$$

where the $\lambda_{i}(i=1,2,3)$ are the eigenvalues of the amplification matrix of the system. If the value of the spectral radius is low on a specific range of frequencies, then the system is submitted to dissipation on this range.

The equation of motion of the mass-spring-damper system without any applied force is given by:

$$
m \ddot{q}+c \dot{q}+k q=0
$$

with $q$ the degree of freedom, $m$ the mass, $k$ the spring's stiffness and $c$ the damper's damping (Figure 6). It can be reformulated by making use of the natural pulsation $\omega^{2}=\frac{k}{m}$ and the structural damping $\varepsilon=\frac{c}{2 \omega m}$ as:

$$
\ddot{q}_{n+1}+2 \varepsilon \omega \dot{q}_{n+1}+\omega^{2} q_{n+1}=0
$$

The system of equations Eqs. (2)-(4), together with the equation of motion Eq. (8), can be transformed into the following matrix form:

$$
\mathbf{E} \mathbf{x}_{n+1}=\mathbf{B} \mathbf{x}_{n}
$$


with $\mathbf{x}_{i}=\left(\begin{array}{lll}q_{i} & h \dot{q}_{i} & h^{2} a_{i}\end{array}\right)^{T}$ and the matrices:

$$
\begin{gathered}
\mathbf{E}=\left(\begin{array}{ccc}
1 & 0 & -\beta \\
0 & 1 & -\gamma \\
\left(1-\alpha_{f}\right) \omega^{2} h^{2} & \left(1-\alpha_{f}\right) 2 \varepsilon \omega h & 1-\alpha_{m}
\end{array}\right) \\
\mathbf{B}=\left(\begin{array}{ccc}
1 & 1 & 0.5-\beta \\
0 & 1 & 1-\gamma \\
-\alpha_{f} \omega^{2} h^{2} & -\alpha_{f} 2 \varepsilon \omega h & -\alpha_{m}
\end{array}\right)
\end{gathered}
$$

The amplification matrix is finally given by:

$$
\mathbf{A}=\mathbf{E}^{-1} \mathbf{B}
$$

In order to avoid inverting the matrix $\mathbf{E}$, the eigenvalues of the amplification matrix $\mathbf{A}$ are obtained knowing that the roots of $\operatorname{det}|\mathbf{A}-\lambda \mathbf{I}|$ are the same as those of $\operatorname{det}|\mathbf{E} \lambda-\mathbf{B}|$ [26]. After calculations, the following characteristic equation which possesses three roots is found:

$$
\begin{array}{r}
\operatorname{det}|\mathbf{E} \lambda-\mathbf{B}|=(\lambda-1)(\lambda-1)\left[\left(1-\alpha_{m}\right) \lambda+\alpha_{m}\right] \\
+\left[\left(1-\alpha_{f}\right) \lambda+\alpha_{f}\right] \omega^{2} h^{2}[\gamma \lambda+(1-\gamma) \\
+(\lambda-1)[\beta \lambda+(0.5-\beta)]] \\
+\left[\left(1-\alpha_{f}\right) \lambda+\alpha_{f}\right] 2 \varepsilon \omega h[\gamma \lambda+(1-\gamma)](\lambda-1)
\end{array}
$$

This equation is then solved in order to study the evolution of the spectral radius with respect to the dimensionless product $\omega h$ for several values of the numerical damping $\rho_{\infty}$ and of the structural damping $\varepsilon$.

\section{V.I Analytical solution}

The amplification matrix A calculated for the generalized$\alpha$ method can be compared to its exact expression $\mathbf{A}^{\text {exact }}$ since the equation of motion Eq. (8) possesses an analytical solution. The goal is then to find $\mathbf{A}^{\text {exact }}$ such that:

$$
\left(\begin{array}{c}
q_{n+1} \\
h \dot{q}_{n+1}
\end{array}\right)=\mathbf{A}^{\text {exact }}(h, \omega, \varepsilon)\left(\begin{array}{c}
q_{n} \\
h \dot{q}_{n}
\end{array}\right)
$$

knowing that the solution of Eq. (8) has the following form if $\varepsilon<1$ :

$$
q_{n+1}=e^{-\varepsilon \omega h}\left(C_{1} \cos \omega_{d} h+C_{2} \sin \omega_{d} h\right)
$$

with the following parameters:

$$
\begin{aligned}
\omega_{d} & =\omega \sqrt{1-\varepsilon^{2}} \\
C_{1} & =q_{n} \\
C_{2} & =\frac{1}{\omega \sqrt{1-\varepsilon^{2}}}\left(\dot{q}_{n}+\varepsilon \omega q_{n}\right)
\end{aligned}
$$

After some calculations, the four components of the matrix $\mathbf{A}^{\text {exact }}$ are found:

$$
\begin{array}{r}
A_{11}^{\text {exact }}=e^{-\varepsilon \omega h}\left[\cos \left(\sqrt{1-\varepsilon^{2}} \omega h\right)+\frac{\varepsilon \sin \left(\sqrt{1-\varepsilon^{2}} \omega h\right)}{\sqrt{1-\varepsilon^{2}}}\right] \\
A_{12}^{\text {exact }}=e^{-\varepsilon \omega h} \frac{\sin \left(\sqrt{1-\varepsilon^{2}} \omega h\right)}{\omega h \sqrt{1-\varepsilon^{2}}} \\
A_{21}^{\text {exact }}=-e^{-\varepsilon \omega h} \frac{\omega h \sin \left(\sqrt{1-\varepsilon^{2}} \omega h\right)}{\sqrt{1-\varepsilon^{2}}} \\
A_{22}^{\text {exact }}=e^{-\varepsilon \omega h}\left[\cos \left(\sqrt{1-\varepsilon^{2}} \omega h\right)-\frac{\varepsilon \sin \left(\sqrt{1-\varepsilon^{2}} \omega h\right)}{\sqrt{1-\varepsilon^{2}}}\right]
\end{array}
$$

In the particular case where the structural damping is equal to $\varepsilon=1$, the solution of the equation of motion is:

$$
q_{n+1}=e^{\varepsilon \omega h}\left(C_{1}+C_{2} h\right)
$$

with the two constants:

$$
\begin{aligned}
& C_{1}=q_{n} \\
& C_{2}=\dot{q}_{n}+\omega q_{n}
\end{aligned}
$$

Thus, the components of the new exact amplification matrix $\mathbf{A}_{\varepsilon=1}^{\text {exact }}$ are the following:

$$
\mathbf{A}_{\varepsilon=1}^{\text {exact }}=e^{-\varepsilon \omega h}\left(\begin{array}{cc}
1+\omega h & 1 \\
\omega h(1-\varepsilon-\varepsilon \omega h) & 1-\varepsilon \omega h
\end{array}\right)
$$

These two matrices are characterised by two eigenvalues. The spectral radius is then given by:

$$
\rho=\max \left(\left|\lambda_{1}\right|,\left|\lambda_{2}\right|\right)
$$

As in the numerical model, its evolution with respect to the dimensionless product $\omega h$ will be studied for several values of the structural damping $\varepsilon$.

\section{V.II Comparison between the analytical and numerical solutions}

In the model of a real structure, the frequency content can be divided into three parts. First, the low frequency domain such that $\omega h \lesssim 0.5$, for which the numerical simulation should be accurate in order to have a good representation of the physical behaviour. It implies that the damping introduced in the model should be a good approximation of the real damping.

Then, the second part is defined by high frequency modes such that $\omega h \gtrsim 2$. For this range of the spectrum, 


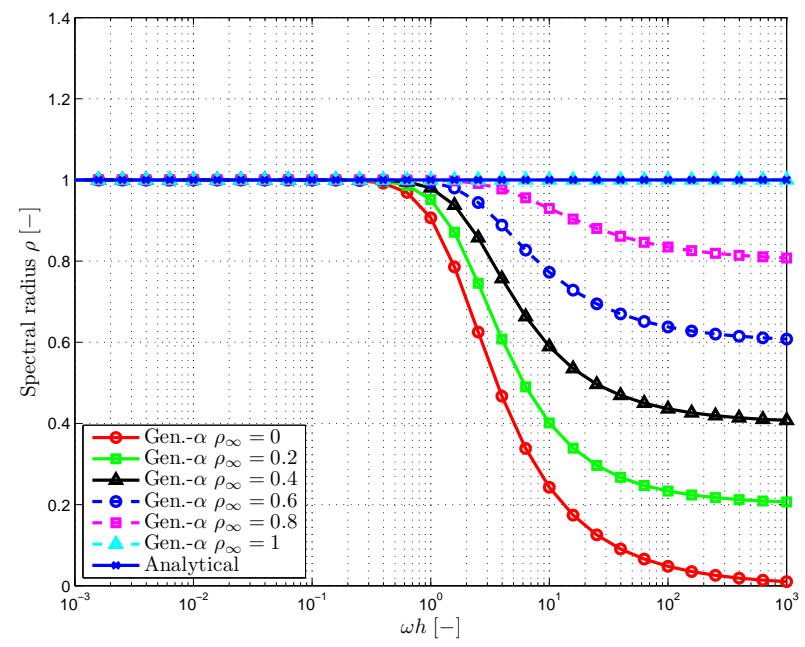

(a) Damping parameters: $\varepsilon=0,0 \leq \rho_{\infty} \leq 1$

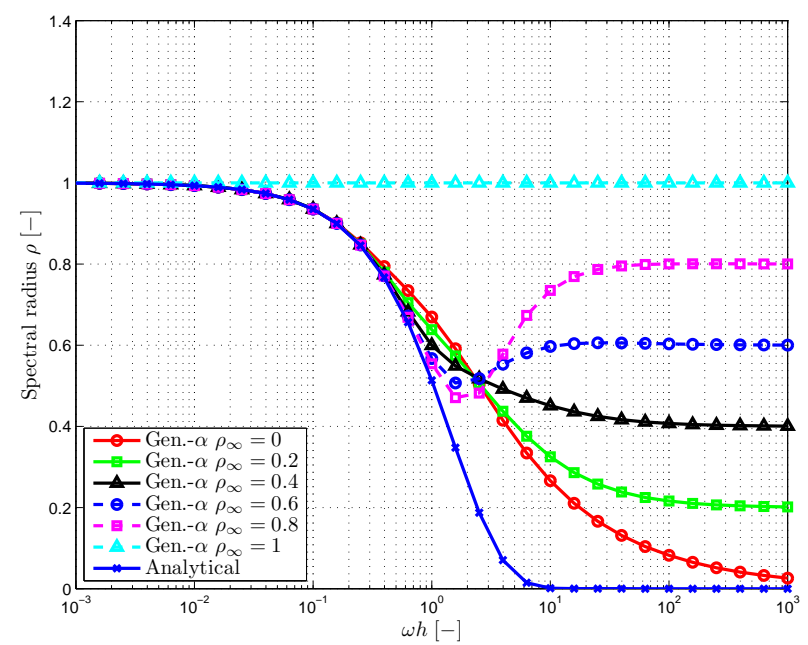

(c) Damping parameters: $\varepsilon=2 / 3,0 \leq \rho_{\infty} \leq 1$.

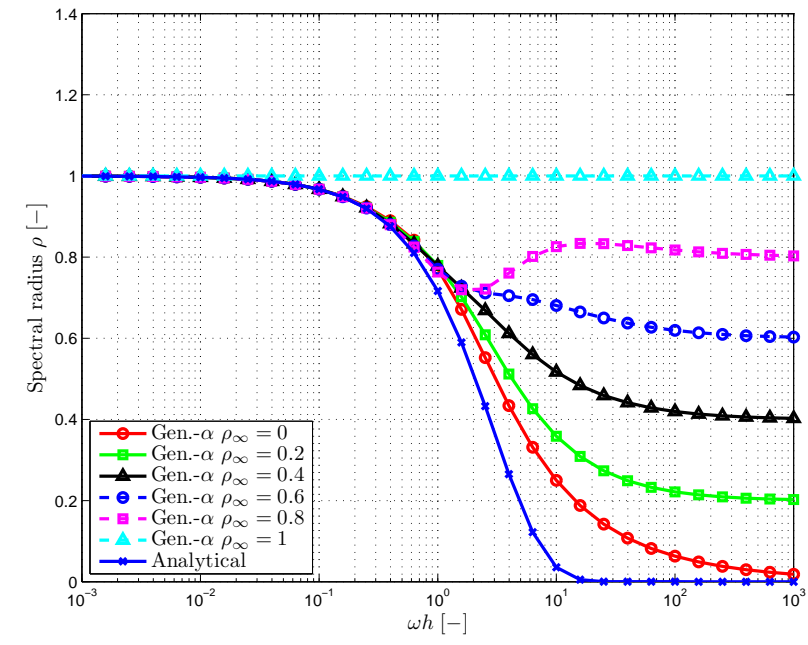

(b) Damping parameters: $\varepsilon=1 / 3,0 \leq \rho_{\infty} \leq 1$

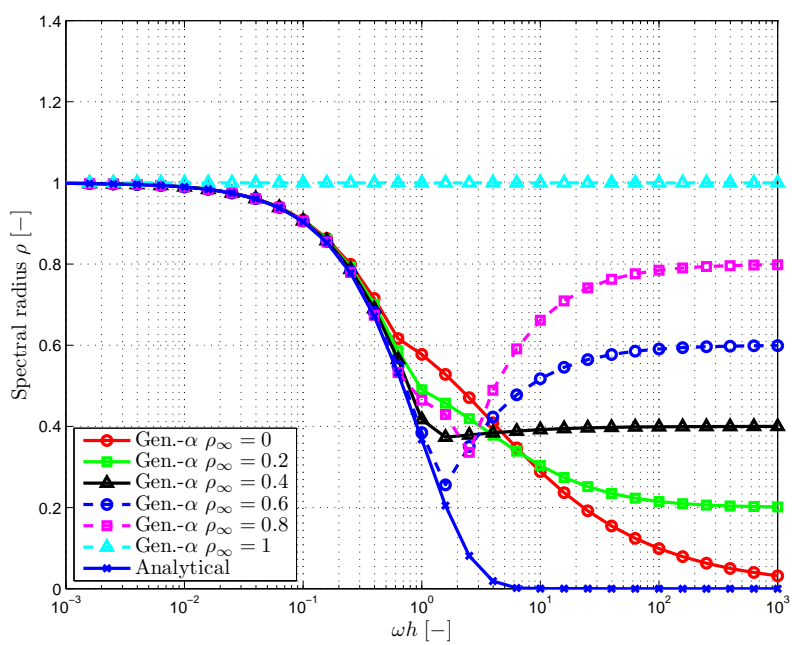

(d) Damping parameters: $\varepsilon=1,0 \leq \rho_{\infty} \leq 1$.

Figure 7: Comparison between the analytical solution of the equation of motion (8) and the resolution with the generalized- $\alpha$ method for several combinations of the damping parameters.

some dissipation must be present for purely numerical reasons. Indeed, it guarantees the convergence of the algorithm and a solution that is unaffected by the poorly represented modes of this domain.

Finally, the last part contains the intermediate frequencies for which a reasonable compromise must be found to ensure a smooth transition between low and high frequency domains.

In order to determine how the evolution of the spectral radius is affected by the structural and numerical damp- ings in the mass-spring-damper system, the analytical and numerical solutions are compared for several combinations of the damping parameters (Figure 7).

It can be seen in Figure $7 \mathrm{a}$ that when no structural damping $(\varepsilon=0)$ is present in the model, the spectral radius stays obviously equal to unity over the whole frequency domain and this behaviour is numerically recovered when there is no numerical damping $\left(\rho_{\infty}=1\right)$. Furthermore, this figure also highlights the effects of performing simulations with numerical damping only. As 
expected, the decrease in the spectral radius is sharper in the high frequency domain when $\rho_{\infty}$ gets closer to zero, leading thus to a higher dissipation of its content, while on the opposite, the low frequency response remains unaffected.

If some structural damping is now introduced, the analytical solution follows an evolution characterised by the three parts mentioned previously (Figures 7b, 7c and 7d): a low frequency domain, a high frequency domain and an intermediate zone. Regarding the numerical curves, they first coincide with the analytical one in the low frequency domain as required. The curves tend to remain superimposed on a larger frequency range when the structural damping is high and the numerical damping is low. On the opposite, in the high frequency domain, they clearly diverge from the analytical solution, especially when the numerical damping is low. Moreover, the curves always converge to the value imposed by the numerical damping $\rho_{\infty}$. Thus, it proves that in the numerical model the structural damping cannot provide the desired dissipation properties on this part of the frequency domain.

It can be observed that the curve related to $\rho_{\infty}=0$ is the only one to converge to the same value as the analytical solution in high frequencies. However, other values of $\rho_{\infty}$ are equally acceptable because accuracy is not sought in this part of the domain. Indeed, the only important factor is the amount of dissipation that is required to ensure the convergence of the solution.

To conclude, in order to have a valid numerical solution, a correct representation of the structural damping is needed in the low frequency range and some numerical damping should be introduced to filter the undesired high frequency spurious modes. These conclusions concern a simple linear problem, while in tape springs, the nonlinearities give rise to more complex behaviours such as couplings and transfers of energy between the low and high frequencies. Nonetheless, it gives a good insight of the importance and the impacts of the structural and numerical dampings.

\section{IMPACT OF THE NUMERICAL DAMPING ON THE DYNAMIC BEHAVIOUR OF TAPE SPRINGS}

In order to determine the impact of the numerical damping on the dynamic behaviour of tape springs, a model is solved several times with different values of $\rho_{\infty}$, but without any structural damping. The pattern described in Section III is followed: first, the tape spring is folded in the opposite sense until the bending angle reaches $60^{\circ}$, then the structure is released and its dynamic behaviour is analysed for $110 \mathrm{~s}$. Furthermore, in order to simplify the behaviour in the equal sense bending and reduce the impact of the torsion, the displacements in the transverse direction are locked on the whole length of the median. The structure consists of a long tape spring, the properties of which are the same as in Table 1, but with a length $L$ of $400 \mathrm{~mm}$. Regarding the boundary conditions, it is clamped on one extremity and rigidly connected to a lumped mass of $0.5 \mathrm{~kg}$ on the other one (Figure 8). The purpose of this lumped mass is to simulate the deployment and the inertia of an appendix.

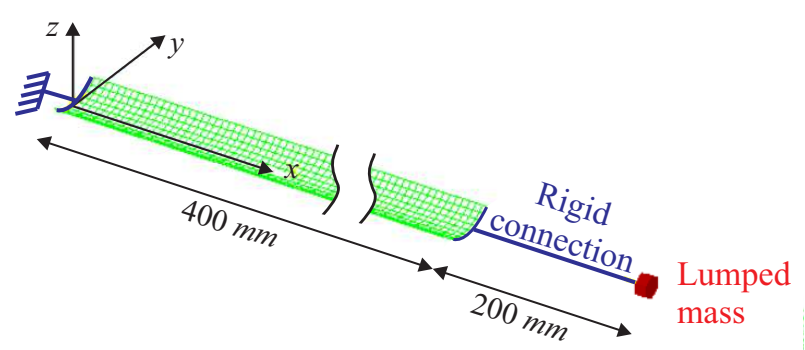

Figure 8: Tape spring with the properties of Table 1 but with a length of $400 \mathrm{~mm}$ rigidly connected to a lumped mass $(0.5 \mathrm{~kg})$.

The evolution of the bending moment for this model with $\rho_{\infty}=0.2$ is given in Figure 9. Notice that in this case the $x$-axis is the elapsed time of the simulation since a dynamic analysis is performed. Nonetheless, as the first part (from 0 to $60 s$ ) is very slow and corresponds to the folding with a constant bending velocity, the time is directly related to the bending angle as in Figure 4.

Furthermore in this part, the numerical damping has a low impact on the value of the peak moment $M_{+}^{\max }$ and the residual moment $M_{+}^{*}$ as it can be seen in the first two plots of Figure 10. However, as it can be expected, a reduction of the numerical damping implies longer periods of oscillations after the buckling around $10.4 s$ since the dissipation in the system is less effective. Thus, Figure 10 (third plot) gives the time at which the difference between the bending moment and the residual moment determined in the second plot of Figure 10 reaches less than $10 \%$. Between the two extreme situations with $\rho_{\infty}=0.2$ and $\rho_{\infty}=0.8$, an increase in time of $24.83 \%$ is noticed. For the latter value, the associated results were obtained after a CPU time of 153 hours, therefore simulations for which $\rho_{\infty}>0.8$ were not performed.

In the strictly dynamic part of the simulation (from 60 to $170 \mathrm{~s}$ ), the deployment of the tape spring leads to large asymmetric oscillations submitted to very low damping whatever the value of $\rho_{\infty}$ (Figure 9). On that same figure, 


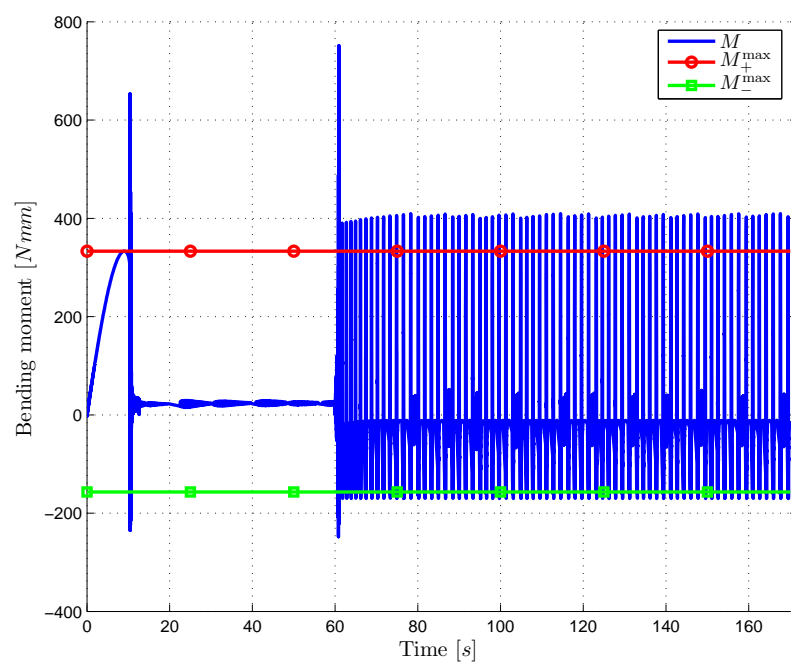

Figure 9: Bending moment evolution for the tape spring of Figure 8 with $\rho_{\infty}=0.2$.
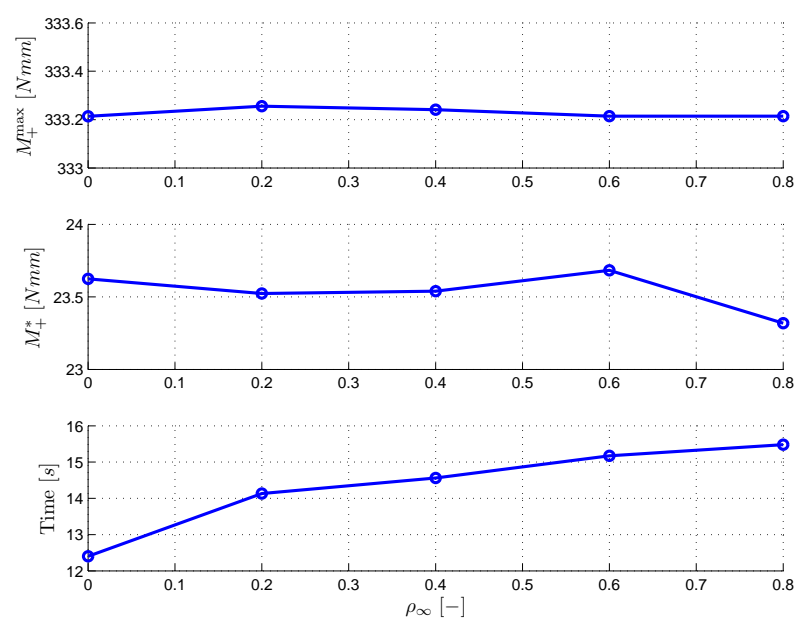

Figure 10: Peak moment $M_{+}^{\max }$, residual moment $M_{+}^{*}$ and duration of the oscillations after buckling for several values of the numerical damping $\rho_{\infty}$.

it can be seen that the limits defined by the peak moments $M_{+}^{\max }$ and $M_{-}^{\max }$ are both exceeded which is due to dynamic effects. In order to check whether a fold is created or not, the evolution of a kinematic parameter is analysed. In terms of displacements, the tip of the tape spring also oscillates asymmetrically around the equilibrium position (Figure 11). It can be seen that the displacements are such that in the opposite sense bending the limit of buckling (red o-curve) is not reached after the deployment, which implies that the hysteresis phenomenon associated with buckling cycles (see Figure 3) does not occur and does not introduce some dissipation in this load case. On the contrary, in the equal sense bending, each oscillation crosses the limit (green $\square$-curve) leading to the formation of a fold.

It can be concluded that when the dissipation in the system is only characterised by numerical damping, the behaviour in the quasi-static analysis is well represented. Furthermore, in the dynamic analysis, the nonlinear oscillations are only slightly damped because they occur in the low frequency range, i.e. out of the range of influence of the numerical damping.

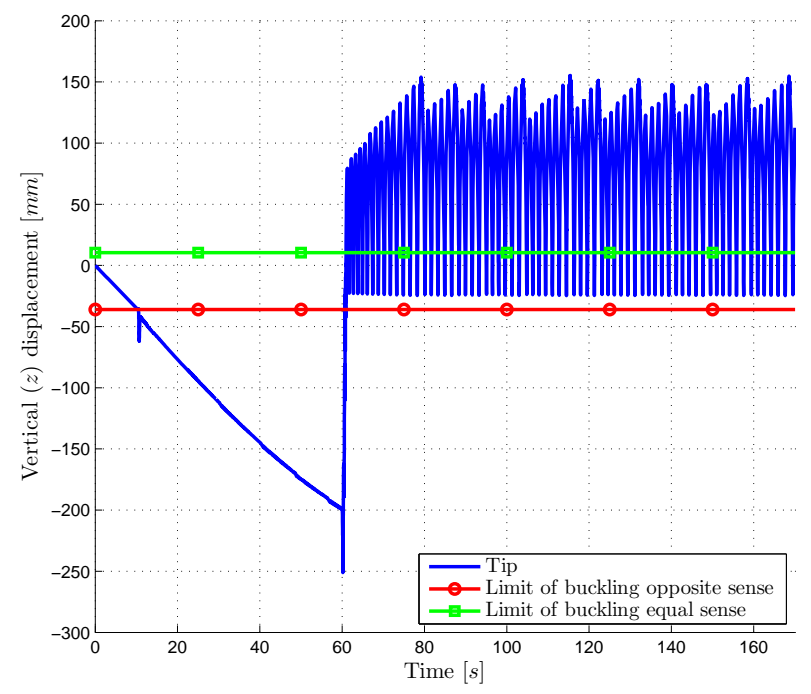

Figure 11: Vertical displacement of the tip of the tape spring with $\rho_{\infty}=0.2$.

\section{INTRODUCTION OF STRUCTURAL DAMPING IN THE TAPE SPRING MODEL}

As it was shown analytically in Section V on a simple one-degree-of-freedom system and numerically in the previous section on a complete tape spring with an appendix, the behaviour of a model cannot be precisely predicted by introducing only numerical damping. Thus, some structural damping is now added in the finite element model described in Figure 8. In order to keep the simulations as simple as possible, the dissipation is represented by Kelvin-Voigt models, which involve a Young modulus $E$ and a viscosity coefficient $\eta$ acting in parallel and correspond to viscoelastic solids [27].

The evolution of the bending moment during the folding and the deployment is given in Figure 12 for $\rho_{\infty}=0.2$ 
and $\eta=10^{-3} s$. Compared to the previous situations without any structural damping, the following elements are noticeable. First, the peak moment $M_{+}^{\max }$ is hardly affected by this new damping (Figure 13, first plot). Then, right after the buckling, the amplitude of the oscillations is much smaller, as well as their duration which is quasi independent from the numerical damping (Figure 14, first plot). During the stabilisation, the residual moment $M_{+}^{*}$ has a better convergence and is also less dependent on the numerical damping (Figure 13, second plot).

Finally, the oscillations after deployment are damped out and, although their duration naturally increases when the numerical damping decreases, the difference between the extreme situations is only of $9.4 \%$ if the oscillations are considered as negligible when they reach $\pm 10 \mathrm{~N} / \mathrm{mm}$. As it was the case in the previous section, the limits defined by the peak moments $M_{+}^{\max }$ and $M_{-}^{\mathrm{max}}$ are both overshot due to dynamic effects, but only folds in the equal sense bending are formed as it can be seen in Figure 15. Furthermore, the dynamic response can be divided into two parts based on the evolution of the amplitudes. First, from 60 to $\sim 80 s$, the oscillations are asymmetric due to the creation of a fold in equal sense bending and they are submitted to a nonlinear damping. Then, from $\sim 80$ to $140 \mathrm{~s}$, the structure does not fold anymore and thus behaves almost linearly. The oscillations are then symmetric with an exponential decrease. If the same zones are analysed in the evolution of the vertical tip displacement of the tape spring (Figure 15), it can be seen that the first part is associated with a sharp reduction of the motion amplitude, followed by an exponential decrease in the second zone. It proves then that the self-locking phenomenon occurs and strongly helps to the stabilisation of the structure in its fully deployed state as expected in experimental applications [7].

\section{CONCLUSIONS}

The purpose of this paper is to determine the impact of the numerical and the structural dampings in a dynamic analysis involving the folding and the deployment of a specific type of compliant structures: tape springs.

First, it is shown on a simple one-degree-of-freedom system that the simulation procedure used to solve the finite element models requires the two types of damping to obtain a valid numerical solution. Indeed, on the one hand, in order to have some dissipation in the high frequency domain, some numerical damping must be introduced. It guarantees the convergence of the algorithm and a solution that is unaffected by the poorly represented

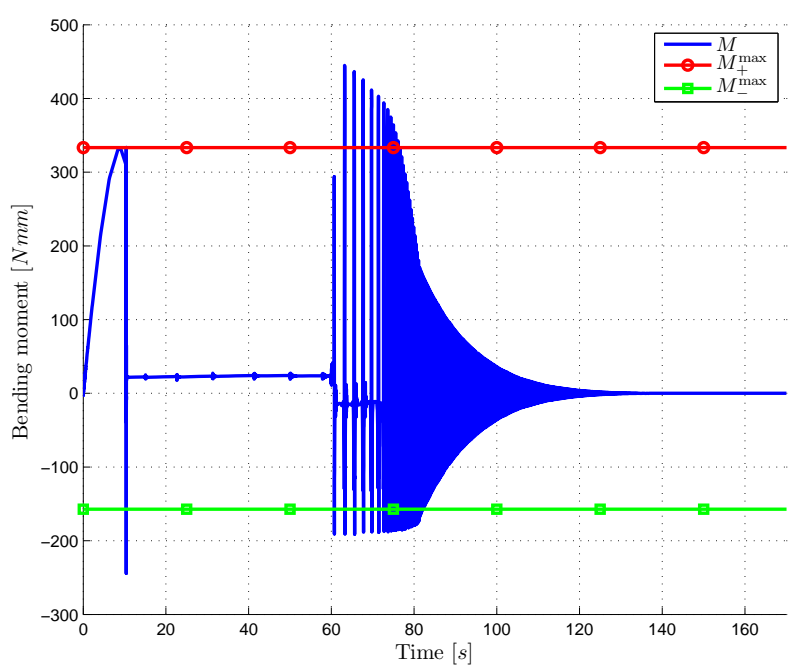

Figure 12: Bending moment evolution for the tape spring of Figure 8 with $\rho_{\infty}=0.2$ and $\eta=10^{-3} s$.
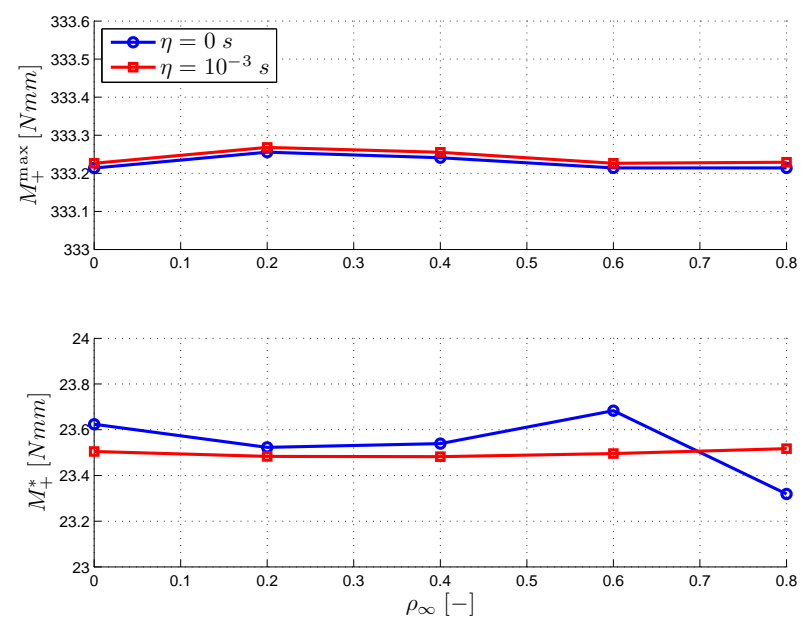

Figure 13: Peak moment $M_{+}^{\max }$ and residual moment $M_{+}^{*}$ for several values of the numerical damping $\rho_{\infty}$.

modes of this domain. Then, on the other hand, regarding the low frequency content, some structural damping is needed in order to have a good representation of the real damping and of the physical behaviour of the system.

Those conclusions are then exploited in a practical case which consists of the finite element model of a long tape spring rigidly connected to an appendix. It is shown that the introduction of structural damping allows reducing the dependence of the characteristic features to the amount of numerical damping and, most importantly, ensures a correct representation of the damping of the oscillations 
after deployment and of the self-locking of the structure in the fully deployed state.
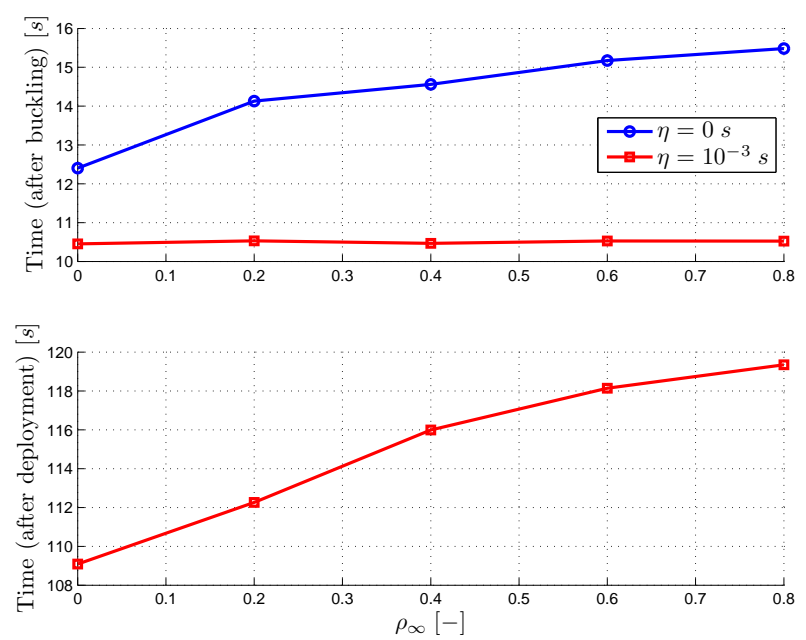

Figure 14: Duration of the oscillations after buckling and after the release of the tape spring for several values of the numerical damping $\rho_{\infty}$.

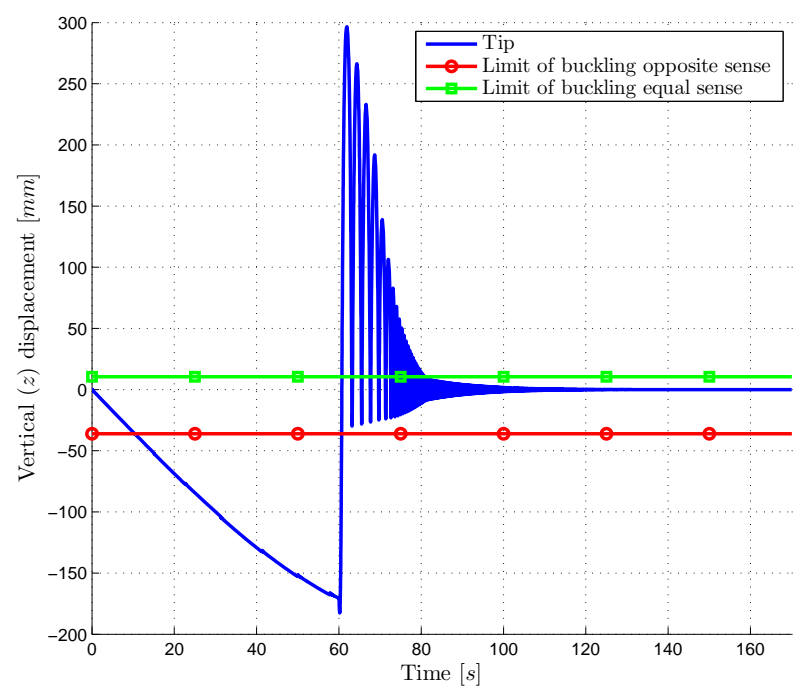

Figure 15: Vertical displacement of the tip of the tape spring with $\rho_{\infty}=0.2$ and $\eta=10^{-3} \mathrm{~s}$.

\section{Acknowledgement}

The first author would like to acknowledge the Belgian National Fund for Scientific Research for its financial support.

\section{References}

[1] S. Pellegrino. Deployable Structures in Engineering. Deployable Structures, CISM courses and lectures 412, Springer, 2001.

[2] K. A. Seffen. Analysis of Structures Deployed by Tape-Springs. Ph. D. thesis, Cambridge University, United-Kingdom, 1997.

[3] W. Wüst. Einige Anwendungen des Theorie der Zylinderschale. Zeitschrift für Angewandte Mathematik und Mechanik, 34, 444-454, 1954.

[4] F. P. J. Rimrott. Querschnittsverformung bei Torsion offener Profile. Zeitschrift für Angewandte Mathematik und Mechanik, 50, 775-778, 1970.

[5] E. H. Mansfield. Large-deflexion torsion and flexure of initially curved strips. Proceedings of the Royal Society of London, Vol. A 334, 1125-1132, 1973.

[6] K. A. Seffen, S. Pellegrino. Deployment of a rigid panel by tape-springs. Department of Engineering, University of Cambridge, Report CUED/DSTRUCT/TR168., 1997.

[7] K. A. Seffen, S. Pellegrino. Deployment Dynamics of Tape-Springs. Proceedings of the Royal Society of London, Vol. A 455, 1003-1048, 1999.

[8] F. Guinot, S. Bourgeois, B. Cochelin, L. Blanchard. A planar rod model with flexible thin-walled crosssections. Application to the folding of tape springs. International Journal of Solids and Structure, Vol. 49 (1), 73-86, 2012.

[9] K. A. Seffen. On the Behaviour of Folded TapeSprings. ASME Journal of Applied Mechanics, Vol. 68, 369-375, 2001.

[10] S. J. I. Walker, G. Aglietti. Study of the Dynamics of Three-Dimensional Tape Spring Folds. AIAA Journal, Vol. 42, 850-856, 2004.

[11] S. J. I. Walker, G. Aglietti. Experimental Investigation of Tape Springs Folded in Three Dimensions. AIAA Journal, Vol. 44, 151-159, 2006.

[12] S. J. I. Walker, G. Aglietti. A study of tape spring fold curvature for space deployable. Proceedings of the Institution of Mechanical Engineers, Part G: Journal of Aerospace Engineering, 313-325, 2007. 
[13] M. Santer, S. Pellegrino. Compliant mutistable structural elements. International Journal of Solids and Structures, Vol. 45, 6190-6204, 2008.

[14] Ö. Soykasap. Analysis of tape spring hinges. International Journal of Mechanical Sciences, Vol. 49, 853-860, 2007.

[15] D. Givois, J. Sicre, . Mazoyer. A low cost hinge for appendices deployment: design, test and application. 9th European Space Mechanisms and Tribology Symposium, Liège, Belgium, 19-21 September 2001.

[16] J. Sicre, D. Givois, E. Emerit. Application of Maeva hinge to myriade microsatellites deployments needs. 11th European Space Mechanisms and Tribology Symposium/ESMATS 2005, ESA/ CNES, Lucerne, Suisse, 21-23 September 2005.

[17] S. Hoffait, O. Brüls, D. Granville, F. Cugnon, G. Kerschen. Dynamic analysis of the self-locking phenomenon in tape-spring hinges. Acta Astronautica, Vol. 66, 1125-1132, 2010.

[18] K. Kwok, S. Pellegrino. Viscoelastic Effects in Tape-Springs. 52nd AIAA/ASME/ASCE/ AHS/ASC Structures, Structural Dynamics and Materials Conference, Denver, CO, 4-7 April 2011.

[19] M. Mobrem, D. Adams. Deployment Analysis of Lenticular Jointed Antennas Onboard the Mars Express Spacecraft. Journal of Spacecraft and Rockets, Vol. 46, 394-402, 2009.

[20] K. A. Seffen, Z. You, S. Pellegrino. Folding and deployment of curved tape springs. International Journal of Mechanical Sciences, Vol. 42, 2055-2073, 2000.

[21] S.A. Samtech, SAMCEF user manual, Version 8.4, 2013.

[22] N. Newmark. A method of computation for structural dynamics. ASCE Journal of the Engineering Mechanics Division, Vol. 85, 67-94, 1959.

[23] H. Hilber, T. Hughes, R. Taylor. Improved numerical dissipation for time integration algorithms in structural dynamics. Earthquake Engineering and Sructural Dynamics, Vol. 5, 283-292, 1977.

[24] J. Chung, G. Hulbert. A time integration algorithm for structural dynamics with improved numerical dissipation: the generalized- $\alpha$ method. ASME Journal of Applied Mechanics, Vol. 60, 371-375, 1993.
[25] C. Calladine. The theory of thin shell structures. Proceedings of the Institution of Mechanical Engineers, Part A: Journal of Power and Energy, Vol. 202, 141-149, 1988.

[26] M. Géradin, A. Cardona. Flexible Multibody Dynamics, A Finite Element Approach. Wiley, 2001.

[27] R. M. Christensen. Theory of Viscoelasticity: An Introduction. Academic Press, 1971. 